\title{
The Targeted Intracellular Delivery of Cytochrome-C Protein to Tumors Using Lipid-Apolipoprotein Nanoparticles
}

\author{
Sang Kyoon Kim, Michael B. Foote, and Leaf Huang
}

\begin{abstract}
Intracellular-acting therapeutic proteins offer a promising clinical alternative to extracellularacting agents, but are limited in efficacy by their low permeability into the cell cytoplasm. We have developed a nanoparticle (NP) composed of lipid (DOTAP/DOPE) and apolipoprotein (APO A-I) to mediate the targeted delivery of intracellular-acting protein drugs to non-small cell lung tumors. NPs were produced with either GFP, a fluorescent model protein, or cytochrome C (cytC), an inducer of apoptosis in cancer cells. GFP and cytC were separately conjugated with a membrane permeable sequence (MPS) peptide and were admixed with DOPE/DOTAP nanoparticle formulations (NPs) to enable successful protein loading. Protein-loaded NPs were modified with DSPE-PEG-Anisamide to enable specific NP targeting to the tumor site in a xenograft model. The resulting particle was $20-30 \mathrm{~nm}$ in size and exhibited a $64-75 \%$ loading efficiency. H460 cells treated with the PEGylated MPS-cytC-NPs exhibited massive apoptosis. When MPS-GFP-NPs or MPS-cytC-NPs were intravenously administered in H460 tumor bearing mice, a specific tumor targeting effect with low NP accumulation in the liver was observed. In addition, MPS-cytC-NP treatment provoked a tumor growth retardation effect in H460 xenograft mice. We conclude that our NP enables targeted, efficacious therapeutic protein delivery for the treatment of lung cancer.
\end{abstract}

\section{Introduction}

Cancer originates from a deficiency or malfunction in somatic proteins participating in cellular homeostasis. Therapies have been produced that treat cancer by silencing abnormal cell signaling patterns using therapeutic proteins as inhibitors. Compared with gene silencing agents, protein drugs have a rapid onset time and are therefore easily controlled by specific dosing. However, manufactured therapeutic proteins are susceptible to proteolysis, denaturation, and aggregation, limiting their efficacy in the body $[1,2]$.

Nanoparticles have been shown to regulate the release of attached proteins based on the degradation behavior of the NP constituent parts [3-5]. If a therapeutic protein is not highly lipophilic, it is difficult to be encapsulated in this system. However, amphiphilic residues, such as membrane permeable sequences (MPS), can be conjugated to non-lipophilic proteins, including cytC or GFP, enabling these proteins to be associated into the lipid bilayer of NPs [6]. A liposomal nanoparticle matrix offers beneficial protection against enzymatic degradation and antibody neutralization, resulting in prolonged retention of

\footnotetext{
() 2012 Elsevier Ltd. All rights reserved.
}

Publisher's Disclaimer: This is a PDF file of an unedited manuscript that has been accepted for publication. As a service to our customers we are providing this early version of the manuscript. The manuscript will undergo copyediting, typesetting, and review of the resulting proof before it is published in its final citable form. Please note that during the production process errors may be discovered which could affect the content, and all legal disclaimers that apply to the journal pertain.

Disclosure Statement

The authors acknowledge that there was no conflict of interest or improper external influence on this work. 
attached protein activity in vivo for as long as the proteins remain complexed to their carriers [7]. Recent research has also determined that nanoparticles conjugated with a cell penetrating peptide (CPP) sequence have shown an increased ability to deliver drug cargo to specific cell types after nonspecific, systematic treatment $[8,9]$.

Nano-size lipid bilayers have previously been developed to study the function of biomembranes [10,11]. Specifically, nanodiscs, or lipid bilayer nanostructures without aqueous internal spaces, have shown potential for lipophilic drug delivery [12-13]. It has been reported that nanodiscs can serve as easily modifiable matrices with a variable affinity for protein drugs dependent on the relative proportions of lipid or introduced functional group constituents [14].

Previously, we have developed several nano-carrier systems to deliver therapeutic cargoes and have studied the protecting and targeting effect of drug carriers surface-modified with target ligands and PEG [15-17]. In the current study, we have documented a delivery platform that creates a nanoparticle constituted by small, refined lipid bilayers of apolipoprotein and DOTAP/DOPE lipid. Our past results indicate that PEGylation and incorporation of anisamide, a ligand that targets the sigma receptor over-expressed in H460 lung carcinoma, on the surface of the nanoparticle can enable successful evasion of RESinduced, non-specific interactions in the liver [18-19]. In this study, we will detail the therapeutic potential of this nanoparticle for delivering MPS-conjugated cytochrome C therapeutic protein into $\mathrm{H} 460$ non-small cell lung carcinoma.

\section{Materials and Methods}

\section{Characterization of MPS conjugated proteins, MPS-GFP-NPs, and MPS-cytC-NPs}

MPS-conjugated protein drugs and NPs were created as described in further detail in the Supplemental Information section. Briefly, MPS peptide (MPS: H-A-A-V-A-L-L-P-A-V-LL-AL-L-A-K-OH, 1548 MW from Anaspec, San Jose, CA) was activated with a 1:2 molar ratio of protein:EDAC, and the resulting solution was dialyzed. GFP or cytC that had been separately incubated with Alexa-488 succinimidyl esters (Invitrogen) was then reacted with the activated MPS in a 1:2 molar ratio for six hours.

The DOTAP/DOPE/apoA-I nanoparticle was produced using a sodium cholate dialysis method $[20,21]$. Small unilamellar liposomes were prepared by mixing DOTAP and DOPE (Avanti Polar Lipids, Inc) dissolved in chloroform in a 2:1 molar ratio (DOTAP:DOPE), evaporating the solvent, then hydrating the mixture with Tris-HCL buffer and reacting the liposomes with ApoA-I in a DOTAP:DOPE:apoA-I molar ratio of either 50:25:1 or 66:33:1. The solution was incubated overnight until transparent and cholic acids were removed [21, 22]. The MPS-GFP or MPS-cytC proteins were then co-incubated with the particles for $1 \mathrm{~h}$ to facilitate protein-NP coupling before PEGylation. The MPS-cytC loading efficiency in the NPs was measured using a Sepharose CL-6B column (Amersham Biosciences, Uppsala, Sweden). DSPE-PEG-anisamide was synthesized in our lab as described elsewhere [16, 19]. Targeted NPs were prepared by incubating the NP solution $(238 \mu \mathrm{L})$ with a $15 \%$ micellar solution of a 1:1 mixture of DSPE-PEG 2000 and DSPE-PEG $_{2000}$-anisamide $(10 \mathrm{mg} / \mathrm{mL})$, at $50{ }^{\circ} \mathrm{C}$ for $10 \mathrm{~min}$.

\section{Flow cytometry assay for detection of apoptosis}

Estimation of apoptotic NCI-H460 lung cancer cell subpopulations was determined using Annexin V-FITC and PI. Briefly, $1 \times 10^{5}$ cells per sample were washed with PBS, and then were incubated in calcium binding buffer with $3 \mu \mathrm{L}$ of Annexin V-FITC $(0.5 \mathrm{mg} / \mathrm{mL})$ for 20 min in darkness. Cells were washed with PBS, were suspended in $500 \mu \mathrm{L}$ of calcium binding buffer, then propidium iodide (PI) $(5 \mathrm{mg} / \mathrm{mL})$ was added and the samples were 
immediately analyzed by flow cytometry. All flow cytometric analyses were performed on a BD FACS Canto ${ }^{\mathrm{TM}}$ flow cytometer (BD, RUO special order system, CA). A $20 \mathrm{~mW}$ blue laser emitting a 488-nm beam served as the excitation source for Annexin V-FITC (FL1) and PI (FL2). Annexin V (green) and PI (red) fluorescent signals were collected with 530 $\mathrm{nm}$ and $610 \mathrm{~nm}$ band pass filters, respectively, for 10,000 cells per sample.

\section{Cell imaging by confocal microscopy}

NCI-H460 cells were fixed with $2 \%$ paraformaldehyde after a $6 \mathrm{~h}$ exposure to Alexa488conjugated MPS-cytC-NPs and a subsequent $1 \mathrm{~h}$ incubation with MitoRed (red). Nuclei were counterstained with DAPI (Sigma Aldrich, St. Louis, MO). Confocal images were acquired with an SP2 Laser Scanning Confocal Microscope (Leica, Bannockburn, IL).

\section{Tissue distribution and tumor uptake study}

Female nude mice, 5-6 weeks old, were purchased from NCI. H460 xenograft tumor bearing mice (size $40-50 \mathrm{~mm}^{2}$ ) were produced on the $6^{\text {th }}$ day after injection of $5 \times 10^{6} \mathrm{NCI}$ $\mathrm{H} 460$ cells on the rear side of the back of mice. In a preliminary study, mice exhibiting tumors $\sim 0.5 \mathrm{~cm} \times 0.5 \mathrm{~cm}$ in size were i.v. injected with $160 \mu \mathrm{g} / \mathrm{kg}$ of the MPS-GFP or GFP nanoparticles in different formulations. In a separate study, mice exhibiting the same size tumors were i.v. injected with MPS-cytC or cytC NPs in different formulations $(160 \mu \mathrm{g} / \mathrm{kg})$. After $4 \mathrm{~h}$, mice were sacrificed and tissues were collected and imaged by the IVIS ${ }^{\mathrm{TM}}$ Imaging System (Xenogen Imaging Technologies, Alameda, CA). Samples were fixed in a $10 \%$ formaldehyde solution for $2 \mathrm{~h}$ and were then washed with PBS. Each treatment was replicated in three mice to ensure statistical significance. The fluorescence intensity of all organs was calculated from the images using manual ROI in the Live Imaging 3.1 software.

\section{Tumor growth retardation study and immunohistology analysis}

Female nude mice bearing $\mathrm{H} 460$ xenograft tumors (size $40-50 \mathrm{~mm}^{2}$ ) were produced six days after subcutaneous injection of $5 \times 10^{6} \mathrm{NCI}-\mathrm{H} 460$ cells on the rear side of the back of mice. CytC or MPS-cytC in different NP formulations were i.v. injected in each respective treatment group $(160 \mu \mathrm{g} / \mathrm{kg}$, administered every other day $)$. Tumor growth in the treated mice was monitored daily by measuring the diameters of tumor with calipers. The tumor volume was calculated as $(\mathrm{W} 2 \times \mathrm{L}) / 2$, where $\mathrm{W}$ is width and $\mathrm{L}$ is length. All mice were eventually sacrificed by cervical dislocation (after 22 days), and tumor tissues from mice were collected for immunohistological study. Serotological and hematological factors were also evaluated to assess toxicity of the various treatments (Table S1). Samples were fixed in a 10\% formaldehyde solution for $2 \mathrm{~h}$ and were then washed with PBS. Tumor sections were prepared by washing tumor tissue with $70 \%$ ethanol, embedding the tumors in paraffin and deparaffinizing the samples in xylene. The sections were then rehydrated in ethanol and washed in phosphate-buffered saline (PBS). Prepared sections were incubated with a 1:200 dilution of anti-Caspase-3 rabbit serum for $2 \mathrm{~h}$ at $25^{\circ} \mathrm{C}$. HRP-labeled secondary antibody (Santa Cruz biotechnology Inc., CA) was added to the blots at a dilution of 1:500 for 20 min. The sections were co-stained with H\&E stain and were observed with a microscope (Nikon Eclipse, Ti-U, Nikon instrument Inc. TX).

\section{Results \\ NP size and charge characterization}

Lipid nanoparticles (NPs) were produced using DOTAP, DOPE, and apolipoprotein (Fig. 1). Using a Nanosizer, we determined that a 2:1 DOTAP:DOPE molar ratio produced lipid NPs of optimal size $(<100 \mathrm{~nm})$ and highly positive charge $(50-55 \mathrm{mV})$ in an aqueous environment. Nanoparticles consisting of DOTAP/DOPE and apolipoprotein were smaller 
(20-30 nm) and displayed a lower cationic charge of 25 28 mV, attributed to enhanced lipid compaction induced by the lipid binding structure of the apolipoprotein. Either MPS-GFP or MPS-cytC was admixed and attached to the formed NPs during a one hour formulation step. Protein-loaded NPs were then PEGylated with a mixture of DSPE-PEG 2000 and DSPE$\mathrm{PEG}_{2000}$-AA (1:1). The resulting final particles, henceforth designated as "MPS-GFP-NP" or "MPS-cytC-NP" in the text, exhibited a slightly reduced size $(20-30 \mathrm{~nm})$ and a dramatically decreased surface charge $(2-3 \mathrm{mV})$. Nanoparticles produced without DSPE$\mathrm{PEG}_{2000}$ were larger and tended to aggregate. All NPs used in subsequent experiments were produced with a 1:1 ratio of DSPE-PEG 2000 and DSPE-PEG $2000-A A$ unless otherwise explicitly noted.

The NP loading efficiency of MPS-GFP was estimated to be ca. $70 \%$ using a Sepharose CL 6B separating column (Amersham Biosciences, Uppsala, Sweden). Similarly, the NP loading efficiency of MPS-cytC was determined to be ca. 64-75\%. The loading efficiency of unconjugated GFP or unconjugated cytC in the NPs was determined to be negligible.

\section{TEM and SEM imaging}

The NP shape is dependent on the ratio of DOTAP to DOPE and the ratio of HDL apoliprotein to loaded protein. After NPs were admixed with MPS-GFP protein, negative TEM staining analysis confirmed the size of the resulting MPS-GFP-NPs to be 20-30 nm (Fig. 2a). In comparison, MPS-cytC-NPs demonstrated a slightly larger shape (Fig. 2b). MPS-cytC-NPs and MPS-GFP-NPs appeared to be refined in size with a slightly flattened morphology. SEM analysis of the NPs adhered on an agarose gel supporting matrix upholds the notion that MPS-cytC-NPs appear to have a flattened shape (Fig. S1b).

\section{Visualization of MPS-cytC delivery in vitro}

After a $6 \mathrm{~h}$ treatment with Alexa-488-labeled MPS-cytC-NPs, H460 cells were incubated with MitoRed (red fluorescence) for 1 hour to stain cell mitochondria. Figure 3 shows partial surrounding and overlap (yellow) of cell mitochondria (red) by the MPS-cytC protein (green). This result demonstrates that MPS-cytC proteins transported to the tumor cell by PEGylated NPs are active around the mitochondria, indicating successful cytC localization for induction of Caspase-9 dependent apoptotic cascades.

\section{Flow cytometry determination of apoptotic induction}

As shown in Supplemental Figure 2, treatment of H460 cells with unconjugated cytC and free MPS-cytC resulted in cleavage of Caspase-9, indicative of apoptosis. Fluctuations in cellular levels of AIF or Endo G are characteristic of cellular apoptosis induced independently of caspase activity [23, 24]. AIF and Endo G, XIAP, and survivin levels in H460 cell lysates remained constant after MPS-cytC treatment, whereas the levels of cleaved Caspase-3 and cleaved Caspase-9 increased (Fig. S3). This result suggests that free MPS-cytC exhibits a penetrating effect in vitro, thereby inducing apoptosis via intracellular activity.

Using flow cytometry, the expression of annexin $\mathrm{V}$ and the permeation of PI were measured in $\mathrm{H} 460$ cells treated for $24 \mathrm{~h}$ with either PBS, empty NPs, cytC, cytC + NPs, MPS-cytC, or MPS-cytC-NPs (Fig. 4). Apoptosis was quantified by the rate of early (Q4) and late apoptotic cells (Q2). As shown in Table 1, only 1.9\% of control cells treated with PBS exhibited apoptosis. In comparison, apoptosis was induced in $13.4 \%$ and $24.4 \%$ of $\mathrm{H} 460$ cells treated with free MPS-cytC and MPS-cytC-NPs, respectively. Significant apoptosis was not induced by a control cytC+ NP treatment of unconjugated cytC (without MPS) admixed with NPs. 


\section{In vivo fluorescence biodistribution analysis}

Negligible fluorescence was detected in the mouse tumors after $4 \mathrm{~h}$ of treatment with PBS, free GFP, or free MPS-GFP. MPS-GFP delivered by unPEGylated NPs mainly accumulated in the liver. However, treatment with PEG-AA modified MPS-GFP-NPs resulted in a high level of fluorescence intensity in the tumor and a comparatively minor intensity in the liver (Fig. 5a). Confocal microscopy images taken of tumor tissue from mice treated with MPSGFP-NPs also show successful tumor delivery of MPS-GFP (Fig. 5b). H460 xenograft mice were likewise treated with MPS-cytC-NPs, and the biodistribution of the Alexa-488 labeled cytC was visualized and quantified (Fig. 6). Negligible fluorescence intensity was detected in mouse tumors after a $4 \mathrm{~h}$ treatment with PBS or free cytC. Conjugation of cytC to MPS resulted in over a 2-fold increase in the delivery of cytC to tumor tissue compared to the delivery of unconjugated cytC. MPS-cytC-NP treatment resulted in a high level of fluorescence intensity in the tumor and comparatively a minor intensity in the liver.

Mice treated with NPs admixed with unconjugated cytC (cytC + NP-PEG-AA) showed tumor accumulation. In the absence of MPS conjugation, cytC delivery in these mice was probably mediated by non-specific interactions between the respective proteins and the cationic NPs.

\section{Immunohistochemical staining of Caspase-3}

Immunohistochemical results show activated Caspase- 3 expression (brown color) concentrated in tumors from H460 xenograft mice treated with MPS-cytC-NPs (Fig. 7). Activated Caspase-3 expression is characteristic of apoptotic induction, therefore the results demonstrate that MPS-cytC carried by the NPs was effective in initiating tumor cell apoptosis in vivo. Tumor tissues from mice treated with MPS-cytC alone demonstrated a much lower level of activated Caspase-3 compared to tumors treated with MPS-cytC-NPs.

\section{In vivo tumor growth analysis}

We examined whether MPS-cytC-NP treatment provoked anti-tumor growth effects. As shown in Figure 8a, there was no significant change in tumor growth observed in $\mathrm{H} 460$ xenograft mice treated with cytC, cytC $+\mathrm{NPs}$, or empty NPs compared to the PBS-treated control group. In contrast, treatment with MPS-cytC-NPs provoked a significant decrease in tumor growth, while free MPS-cytC treatment resulted in partial tumor growth retardation. The therapeutic success of MPS-cytC-NPs indicates that the NP formulation retains the in vivo protection and AA-targeting advantages of the NP platform without compromising the ability of MPS to convey cytC to tumor cells. Increases in the injection dose of MPS-cytCNPs resulted in an enhanced therapeutic effect (Fig. 8b). In contrast, injection of xenograph mice with $160 \mu \mathrm{g} / \mathrm{kg}$ of free MPS-cytC resulted in the maximal therapeutic effect; further increase in the injection dose did not bring about any improved effect.

\section{Discussion}

Effective protein delivery systems hold great potential for treating cancer. However, challenges hindering the successful delivery of proteins, such as poor blood stability, low cellular permeability and high liver uptake, have limited the clinical progress of protein therapeutics [26]. In addition, therapeutic proteins that successfully penetrate into target cells may become entrapped and degraded within endosomes, preventing release to a targeted cellular compartment [27, 28]. Cell-penetrating peptides (CPPs) have been shown to significantly improve protein delivery efficiency, yet these peptides are susceptible to degradation, especially in the presence of proteases, hampering their use in therapeutic applications. 
Our intracellular delivery platform uses nanosized DOTAP/DOPE and apolipoprotein bilayers to provide an effective system for delivering proteins to specific tissues in vivo. We have determined that a 2:1 mole ratio of DOTAP:DOPE produces small lipid bilayer nanoparticles. DOPE, a neutral helper lipid, plays an especially crucial role in improving protein endosomal escape by adopting a hexagonal phase in the endosome lumen that destabilizes the endosomal membrane [29]. Therefore, we believe that the presence of DOTAP and DOPE in our NP formulation enables the endosomal escape of attached proteins, such as cytC, into the cytoplasm of cancer cells [29-33].

Membrane permeable sequences (MPS) can drive protein drugs to a specific site, an ability enhanced in conjunction with NPs exhibiting a notably strong targeting and protecting effect due to a DSPE-PEG-AA outer coating [34-37]. We have clearly demonstrated that the incorporation of this selective ligand allows our NP platform to enhance tumor targeting and promote greater therapeutic effects in an animal model.

With these surface modifications, the NP shape is highly dependent on external conditions, such as loaded protein drugs. MPS-cytC-NPs exhibit a different shape than MPS-GFP-NPs (Fig. 2), an indication that properties such as NP size, net charge, 3D structure and the presence of loaded protein are important determinants of the NP shape.

MPS-cytC-NP treatment both induced apoptosis in $\mathrm{H} 460$ lung cancer cells and prevented H460 tumor growth in vivo. As shown by the activated Caspase-9 western blots from MPScytC treated $\mathrm{H} 460$ cells (Fig. S2) and the localization of the MPS-cytC-NPs proximal to H460 cell mitochondria (Fig. 3), this result can be explained by the interaction of cytC and apoptosis-inducing factors in the outer mitochondrial membrane of the tumor cells. Previously, cytC has been shown to act on the exterior cell structure to decrease apoptosis and inflammatory reactions related to rheumatoid arthritis [38]. Our study, however, demonstrates massive $\mathrm{H} 460$ tumor cell apoptosis correlated with mitochondrial localization of fluorescent MPS-cytC after MPS-cytC-NP treatment, demonstrating indirect proof of successful NP uptake and MPS-cytC delivery to the tumor cell interior.

Fluorescent tissue distribution data confirms the selective targeting of NPs containing MPSGFP or MPS-cytC to in vivo tumor sites. Once more, intracellular visualization of the MPScytC or MPS-GFP delivered by the NPs indicates successful endosomal escape and localization of the proteins in the cytosol of cancer cells. Importantly, selective targeting is dependent on the co-incubation of the MPS-conjugated therapeutic protein with our NPs. When mice were treated with MPS-GFP or MPS-cytC admixed with un-PEGylated NPs, very strong protein signals were observed in the liver (Figures 5, 6). This result is probably due to the non-specific uptake of unPEGylated NPs by the RES system. In vivo administration of PEGylated NPs, in comparison, resulted in a longer stability effect and a lower liver accumulation effect, delivering the loaded protein drug to tumor cells. Overall, in vivo distribution data clearly demonstrates that the NP formulation can deliver detectable therapeutic proteins at high efficiency while maintaining low toxicity, suggesting potential applications in imaging as well as therapy.

NPs admixed with unconjugated-cytC were partially distributed to xenograph tumors in vivo, whereas free cytC showed little tumor accumulation (Figure 6). One explanation for this result is that charge-charge interactions between cytC and the NP assist in successful tumor delivery in the absence of MPS. Treatment of xenograph mice with cytC admixed with the NPs, however, resulted in 30\% lower tumor accumulation levels than MPS-cytC admixed with NPs. Protein delivery enabled by non-specific interactions between a protein and its carrier is generally not stable; therefore unconjugated-cytC carried by the NPs would exhibit a less dramatic cellular uptake or a lower therapeutic effect in vivo. Indeed, the 
transported cytC did not provoke a tumor retardation effect similar to the effect observed in mice treated with MPS-cytC-NPs (Fig. 8a). This indicates that the force linking unconjugated-cytC and the NP was not strong enough to enable their co-uptake into tumor cells.

\section{Conclusions}

We have demonstrated the successful production of a nanoparticle platform utilizing DOTAP/DOPE lipids and an MPS transporting molecule to specifically deliver therapeutic proteins into tumor tissue. The NP is highly soluble and effective in vitro and in vivo, enabling the successful delivery of a soluble cytC therapeutic protein. Protein drugs loaded to the NP can avoid aggregation, which normally results from protein-protein interactions in vivo. The NPs can be modified with external ligands or protective materials to enhance targeting and maintain biological stability in vivo. Designed NPs are able to avoid excessive RES uptake by the liver, deliver cytC protein into cancer cells, moderate protein escape from endosomal vesicles, and induce apoptosis and tumor growth retardation in vivo. Our NP promises to be a potent tool for the effective delivery of non-lipophilic therapeutic proteins for cancer therapy.

\section{Acknowledgments}

Special thanks goes to Dr. Yuhua Wang for material preparation.

Role of the funding source

This work was financially supported by NIH grants CA129835, CA149363 and CA151652. The NIH did not have any role in the planning or the administration of the research.

\section{References}

1. Tabata Y, Ikada Y. Protein release from gelatin matrices. Adv Drug Deliv Rev. 1998; 31:287-301. [PubMed: 10837630]

2. Manning M, Chou D, Murphy B, Payne R, Katayam D. Stability of protein pharmaceuticals: an update. Pharmaceutical Research. 2010; 27:544-575. [PubMed: 20143256]

3. Porjazoska A, Goracinova K, Mladenovska K, Glavas M, Simonovska M, Janjević EI, et al. Poly(lactide-co-glycolide) microparticles as systems for controlled release of proteins: preparation and characterization. Acta Pharm. 2004; 54:215-229. [PubMed: 15610618]

4. Panyam J, Dali MM, Sahoo SK, Ma W, Chakravarthi SS, Amidon GL, et al. Polymer degradation and in vitro release of a model protein from poly(D, L lactide-co-glycolide) nano- and microparticles. J Control Release. 2003; 92:173-187. [PubMed: 14499195]

5. Mohamed F, Van der Walle CF. Engineering biodegradable polyester particles with specific drug targeting and drug release properties. J Pharm Sci. 2008; 97:71-87. [PubMed: 17722085]

6. Bartz R, Fan H, Zhang J, Innocent N, Cherrin C, Beck SC, et al. Effective siRNA delivery and target mRNA degradation using an amphipathic peptide to facilitate $\mathrm{pH}$-dependent endosomal escape. Biochem J. 2011; 435:475-487. [PubMed: 21265735]

7. Mohanraj VJ, Chen Y. Nanoparticles - a review. Trop J Pharm Res. 2006; 5:561-573.

8. Torchilin VP. Tat peptide-mediated intracellular delivery of pharmaceutical nanocarriers. Adv Drug Deliv Rev. 2008; 60(4-5):548-558. [PubMed: 18053612]

9. Helin R, Pille S, Margus P. Peptide-mediated protein delivery—which pathways are penetrable. Biochimica et Biophysica Acta. 2010; 1798:2240-2248. [PubMed: 20170627]

10. Andrew JL, Timothy HB, Alexander NB, Barry AS, Stephen GS. Functional reconstitution of $\beta 2-$ adrenergic receptors utilizing self-assembling nanodisc technology. BioTechniques. 2006; 40:601612. [PubMed: 16708760] 
11. Denisov IG, Grinkova YV, Lazarides AA, Sligar SG. Directed self-assembly of monodisperse phospholipid bilayer nanodiscs with controlled size. J Am Chem Soc. 2004; 126(11):3477-3487. [PubMed: 15025475]

12. Tufteland M, Pesavento JB, Bermingham RL, Hoeprich PD Jr, Ryan RO. Peptide stabilized amphotericin B nanodisks. Peptides. 2007; 28:741-746. [PubMed: 17293004]

13. Nelson KG, Bishop J, Ryan RO, Titus R. Nanodisk-associated amphotericin B clears Leishmania major cutaneous infection in susceptible BALB/c mice. Antimicrob Agents Chemother. 2006; 50:1238-1244. [PubMed: 16569834]

14. Redmond KA, Nguyen TS, Ryan RO. All-trans-retinoic acid nanodisks. Int J Pharm. 2007; 339(12):246-250. [PubMed: 17412536]

15. Lee KY, Yuk SH. Polymeric protein delivery systems. Prog Polym Sci. 2007; 32:669-697.

16. Li SD, Huang L. Stealth nanoparticles: high density but sheddable PEG is a key for tumor targeting. J Control Release. 2010; 145(3):178-181. [PubMed: 20338200]

17. Li SD, Chen YC, Hackett MJ, Huang L. Tumor-targeted delivery of siRNA by self-assembled nanoparticles. Mol Ther. 2008; 16:163-169. [PubMed: 17923843]

18. Banerjee R, Tyagi P, Li S, Huang L. Anisamide-targeted stealth liposomes: a potent carrier for targeting doxorubicin to human prostate cancer cells. Int J Cancer. 2004; 112(4):693-700. [PubMed: 15382053]

19. Li SD, Huang L. Nanoparticles evading the reticuloendothelial system: role of the supported bilayer. Biochimica et Biophysica Acta (BBA)-Biomembranes. 2009; 1788:2259-2266.

20. Pullerits R, Bokarewa M, Jonsson IM, Verdrengh M, Tarkowski A. Extracellular cytochrome $c$, a mitochondrial apoptosis-related protein, induces arthritis. Rheumatology. 2005; 44(1):32-39. [PubMed: 15367748]

21. Masakazu M, Yoko T, Tetsurou H, Minoru N. Static and dynamic characterization of nanodiscs with apolipoprotein A-I and its model peptide. J Phys Chem B. 2010; 114:12376-12382. [PubMed: 20812712]

22. Bayburt TH, Sligar SG. Membrane protein assembly into nanodiscs. FEBS Letters. 2010; 584:1721-1727. [PubMed: 19836392]

23. Sean PC, Valina LD, Ruth SS. Role of AIF in caspase-dependent and caspase-independent cell death. Oncogene. 2004; 23:2785-2796. [PubMed: 15077142]

24. Ishitsuka K, Hideshima T, Hamasaki M, Raje N, Kumar S, Podar K, et al. Novel inosine monophosphate dehydrogenase inhibitor VX-944 induces apoptosis in multiple myeloma cells primarily via caspase-independent AIF/Endo G pathway. Oncogene. 2005; 24(38):5888-5896. [PubMed: 15940263]

25. Nesrin Ö, Wafik S. Defining characteristics of types I and II apoptotic cells in response to TRAIL. Neoplasia. 2002; 4(6):551-557. [PubMed: 12407450]

26. Brown LR. Commercial challenges of protein drug delivery. Expert Opin Drug Deliv. 2005; 2(1): 29-42. [PubMed: 16296733]

27. Straubinger RM, Hong K, Friend DS, Papahadjopoulos D. Endocytosis of liposomes and intracellular fate of encapsulated molecules: encounter with a low $\mathrm{pH}$ compartment after internalization in coated vesicles. Cell. 1983; 32:1069-1079. [PubMed: 6404557]

28. Huwyler J, Yang J, Pardridge WM. Receptor mediated delivery of daunomycin using immunoliposomes: pharmacokinetics and tissue distribution in rats. J. Phamacol. Exp. Ther. 1997; 282:1541-1546.

29. Talbot WA, Zheng LX, Lentz BR. Acyl chain unsaturation and vesicle curvature alter outer leaflet packing and promote poly(ethylene glycol)-mediated membrane fusion. Biochemistry. 1997; 36(19):5827-5836. [PubMed: 9153423]

30. Felgner PL, Gadek TR, Holm M, Roman R, Chan HW, Wenz M, et al. Lipofection: a highly efficient, lipid-mediated DNA-transfection procedure. Proc Natl Acad Sci USA. 1987; 84:74137417. [PubMed: 2823261]

31. Wasungu L, Hoekstra D. Cationic lipids, lipoplexes and intracellular delivery of genes. J Control Release. 2006; 116:255-264. [PubMed: 16914222]

32. Wrobel I, Collins D. Fusion of cationic liposomes with mammalian cells occurs after endocytosis. Biochim Biophys Acta. 1995; 1235:296-304. [PubMed: 7756338] 
33. Hafez IM, Cullis PR. Roles of lipid polymorphism in intracellular delivery. Adv Drug Deliv Rev. 2001; 47:139-148. [PubMed: 11311989]

34. Popot JL. Amphipols, nanodiscs, and fluorinated surfactants: three nonconventional approaches to studying membrane proteins in aqueous solutions. Annu. Rev. Biochem. 2010; 79:737-775. [PubMed: 20307193]

35. Nath A, Atkins WM, Sligar SG. Applications of phospholipid bilayer nanodiscs in the study of membranes and membrane proteins. Biochemistry. 2007; 46:2059-2069. [PubMed: 17263563]

36. Katzen F, Fletcher JE, Yang JP, Kang D, Peterson TC, Cappuccio JA, et al. Insertion of membrane proteins into discoidal membranes using a cell-free protein expression approach. J. Proteome Res. 2008; 7:3535-3542. [PubMed: 18557639]

37. Alami M, Dalal K, Lelj-Garolla B, Sligar SG, Duong F. Nanodiscs unravel the interaction between the Sec YEG channel and its cytosolic partner SecA. EMBOJ. 2007; 26:1995-2004.

38. Abhinav N, Atkins WM, Sligar SG. Applications of phospholipid bilayer nanodiscs in the study of membranes and membrane proteins. Biochemistry. 2007; 46(8):2059-2069. [PubMed: 17263563] 

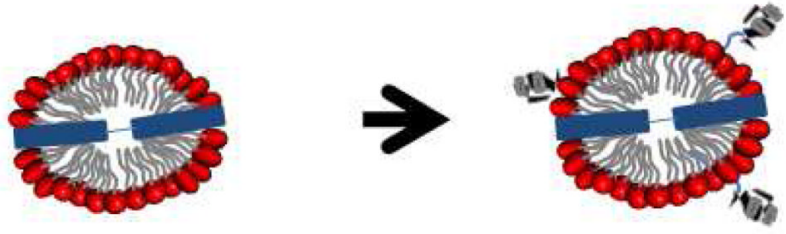

Protein loading
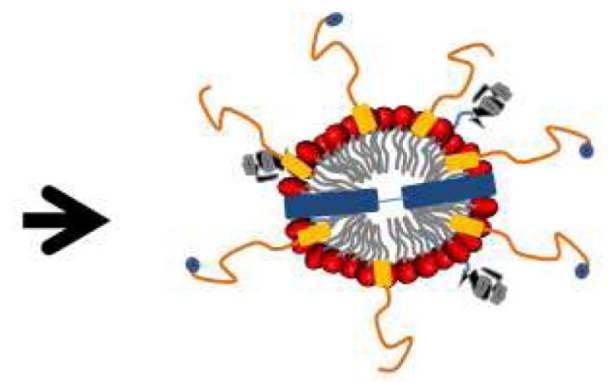

NP formation $\sim$ DOTAP: DOPE $(1: 0.5)$

Apolipoprotein $\sim:$ A-A-V-A-L-L-P-A-V-L-L-A-L-L-A-K (MPS) : protein therapeutic
PEGylation of the NP surface

\section{Figure 1.}

Schematic picture depicting our NP manufacturing platform for protein delivery. 
(a)

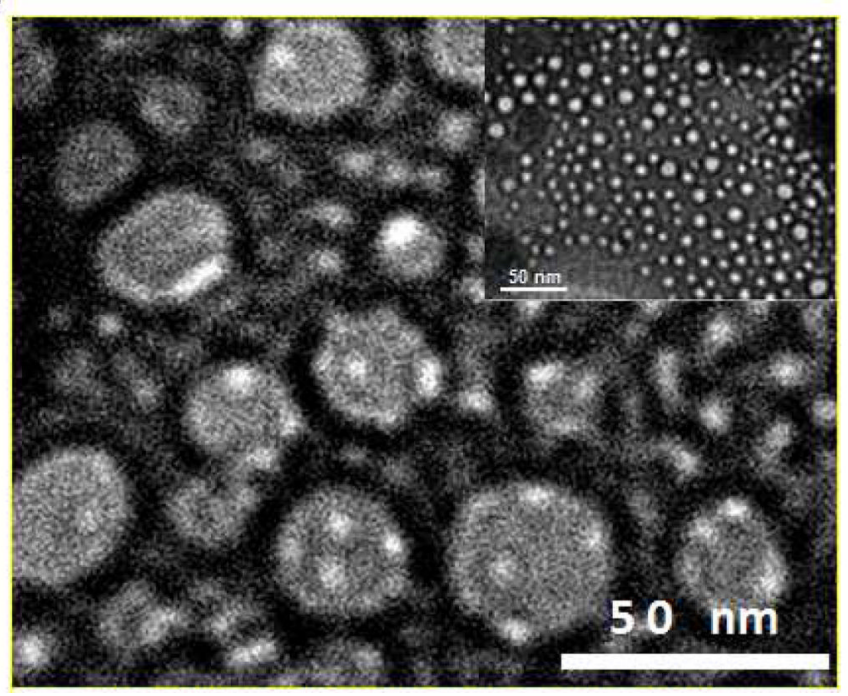

(b)

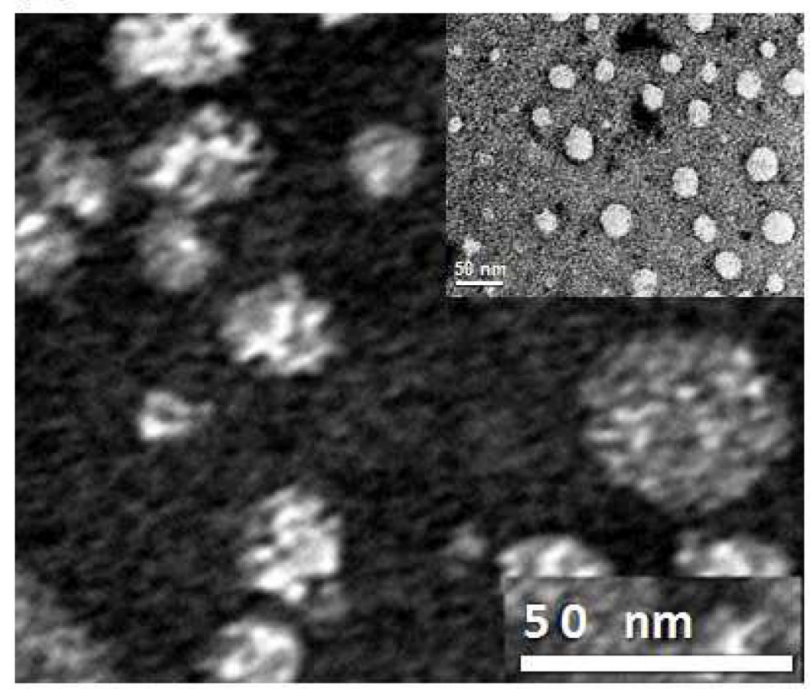

Figure 2.

TEM images of PEGylated NPs admixed with MPS-GFP (a) and MPS-cytC (b). 


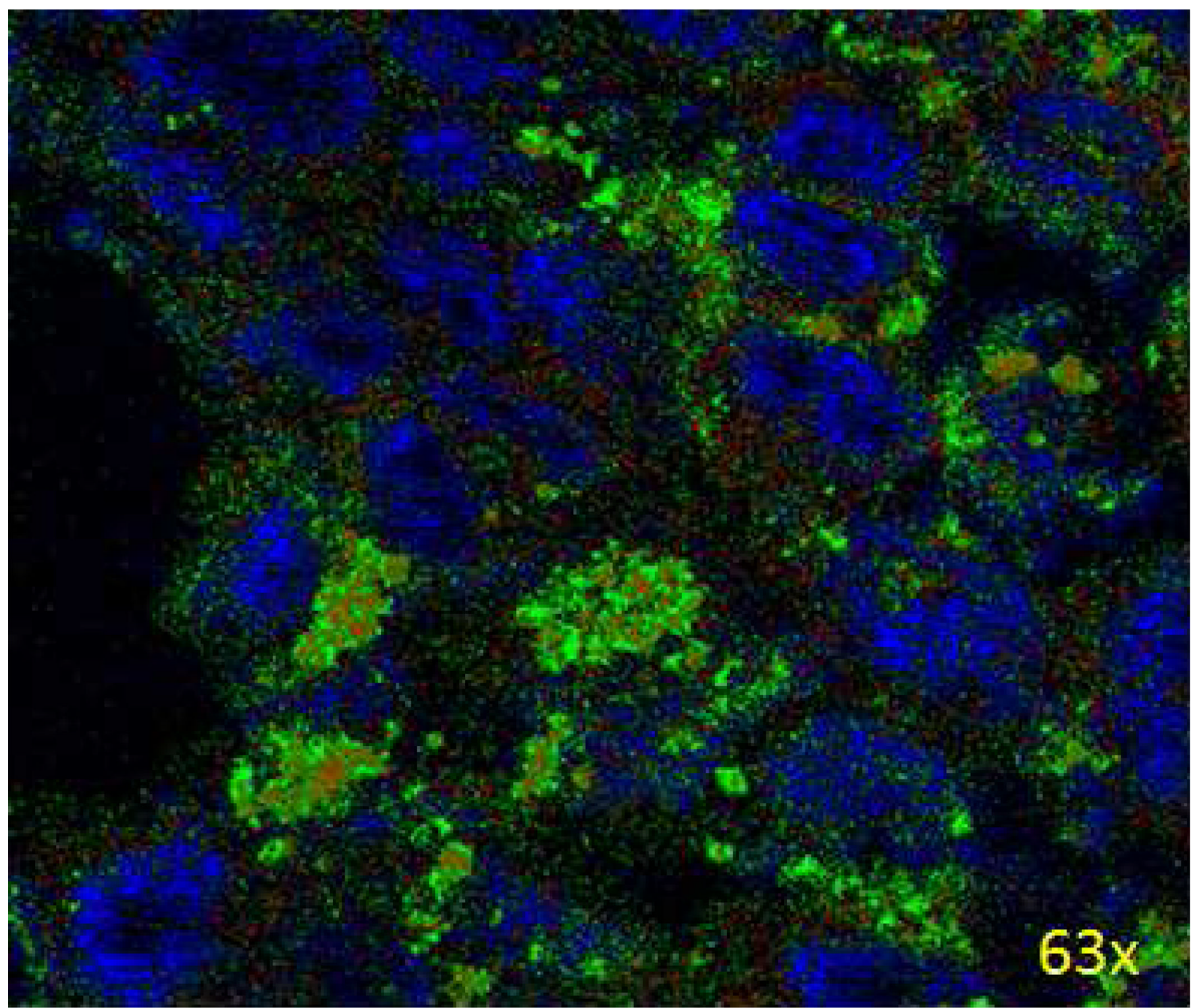

Figure 3.

Uptake and intracellular accumulation in $\mathrm{H} 460$ cells after $6 \mathrm{~h}$ incubation with Alexa 488labeled MPS-cytC-NPs (green) measured using confocal microscopy. Cell nuclei were stained with DAPI (blue) and cell mitochondria were stained with MitoRed (red). 

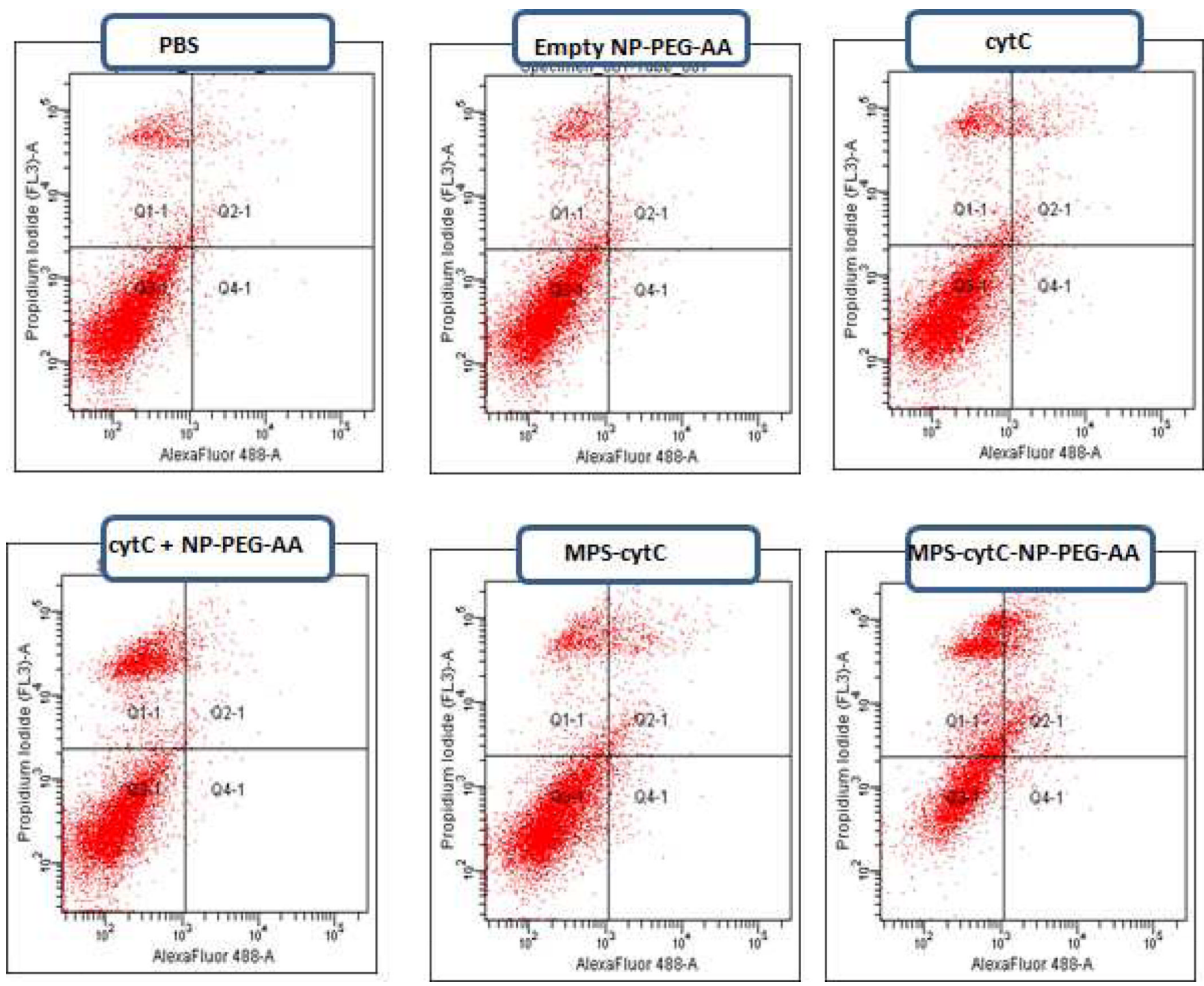

Figure 4.

Apoptotic induction as detected by Annexin V (x axis) and PI (y axis) staining of H460 cells after $12 \mathrm{~h}$ treatment with different formulations. The percentage of cells in the Q2 and Q4 quadrants is shown in Table 1 and is expressed as mean \pm SEM $(\mathrm{N}=3)$ 


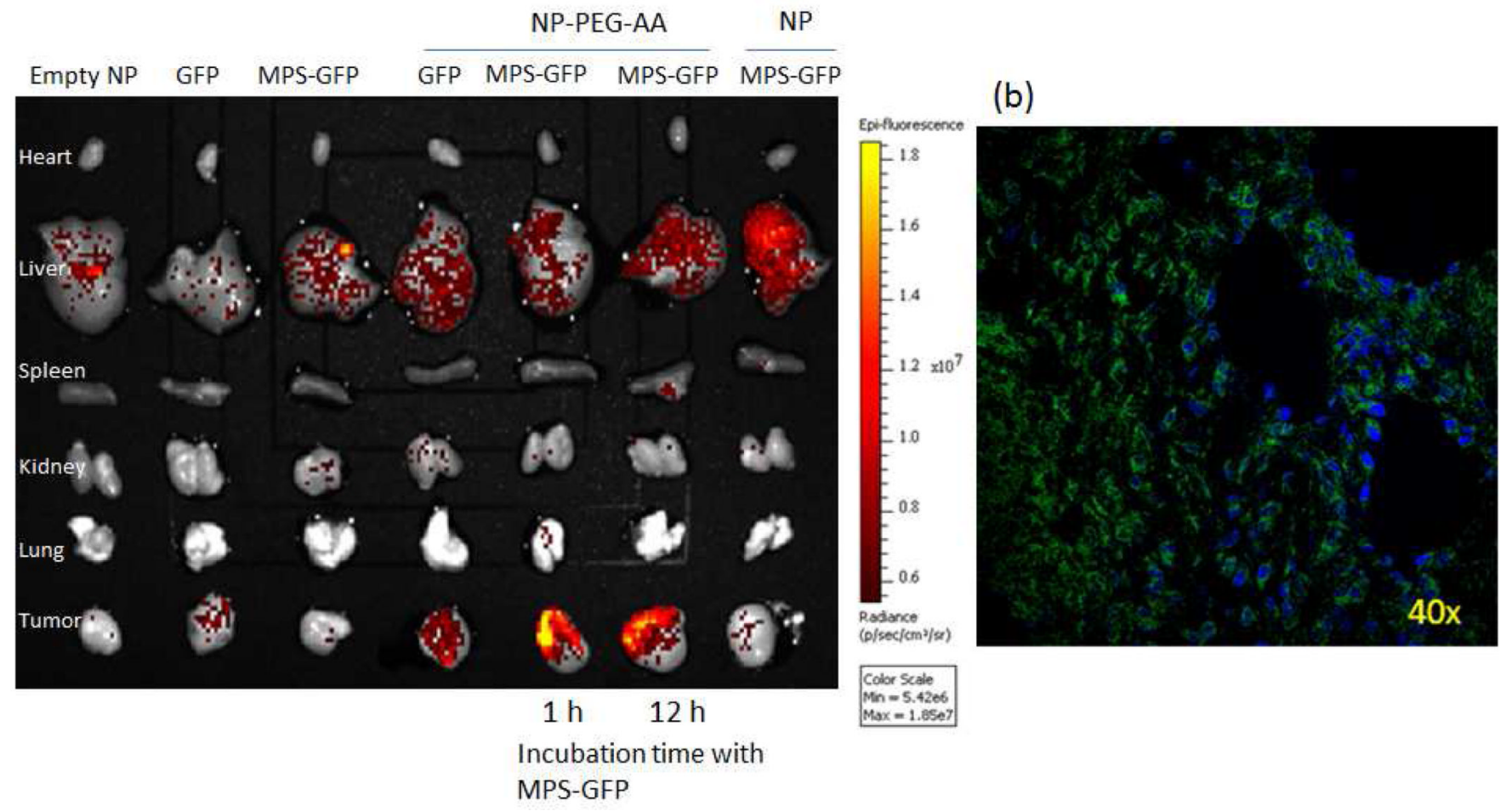

Figure 5.

Distribution of intravenously administered free GFP, free MPS-GFP, GFP + NP, and MPSGFP-NP in major organs (heart, liver, spleen, kidney, lung, tumor) of H460 xenograft mice imaged using a Xenogen IVIS imaging system. GFP-MPS-NPs were prepared with or without DSPE-PEG/DSPE-PEG-AA as indicated. A control mouse group was treated with empty NP-PEG-AA. Organs were imaged using a kinetic IVIS optical imaging program v 3.1 (a). Confocal microscopy images were taken of tumor tissue from mice treated with MPS-GFP-NP-PEG-AA (b). Blue coloring indicates DAPI nuclear staining while green coloring represents GFP expression. 
(a)
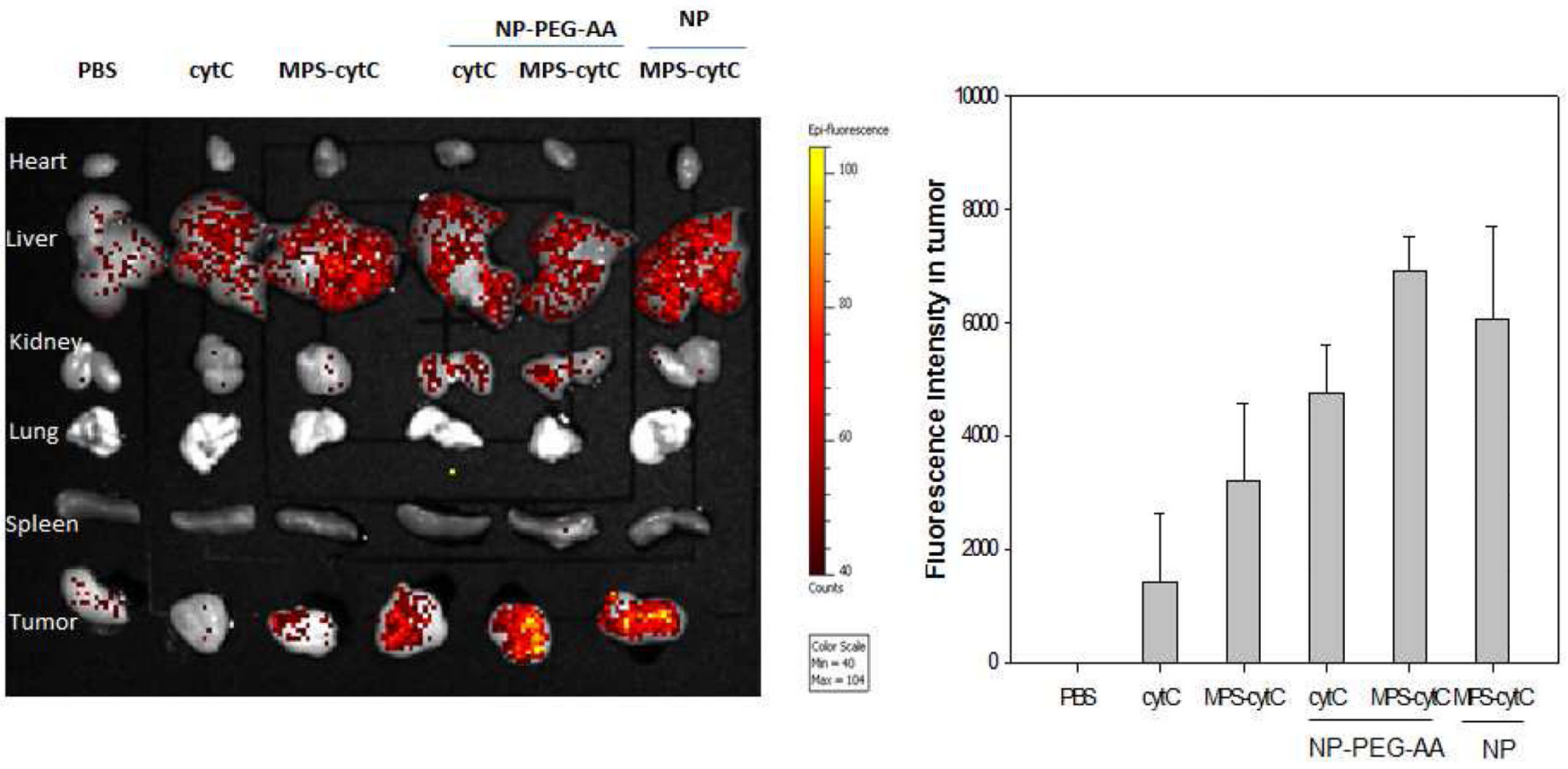

Figure 6.

Distribution of intraveneously administered Alexa-488 labeled cytC, cytC + NP, or MPScytC-NPs in major organs (heart, liver, kidney, lung, spleen, tumor) of H460 xenograft mice imaged using a Xenogen IVIS imaging system. NP formulations were prepared with or without DSPE-PEG/DSPE-PEG-AA as indicated. Empty NP-PEG-AA, free cytC and free MPS-cytC served as control treatments. Organs were imaged (a) and quantified (b) using a kinetic IVIS optical imaging program v 3.1. Error bars indicate the Standard Deviation of the data. 

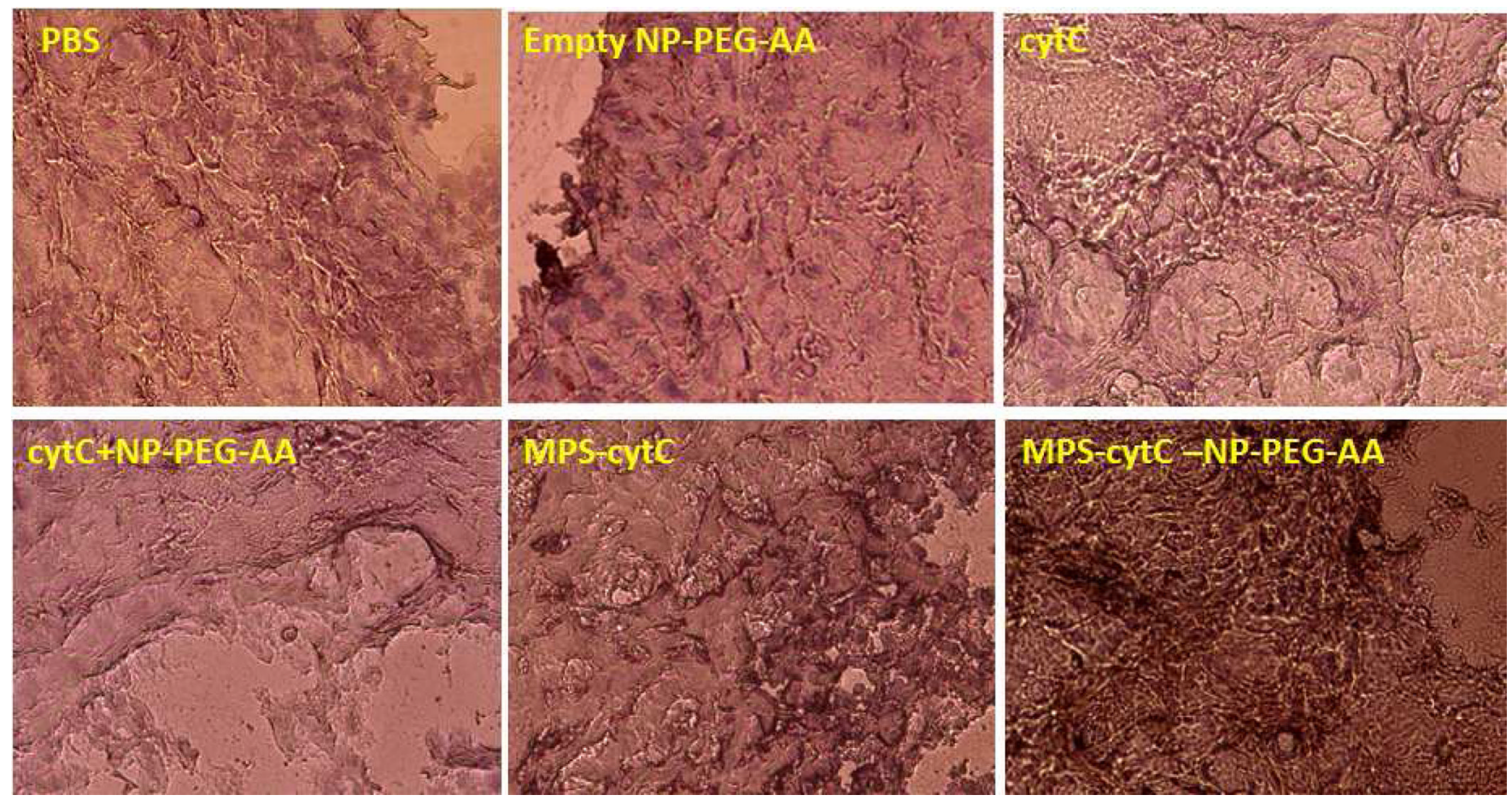

Figure 7.

Localization of activated Caspase- 3 by immunohistochemistry in the tumor sections of mice treated with PBS (a), empty NP (b), cytC (c), cytC + NP-PEG-AA (d), MPS-cytC (e), and MPS-cytC-NP-PEG-AA (f). All tissues were stained with Caspase-3 antibody (brown staining). 



Figure 8.

Tumor growth retardation effect of different formulation treatments in an $\mathrm{H} 460$ xenograft mouse model. CytC $(40 \mu \mathrm{g} / \mathrm{kg})$ or MPS-cytC $(160 \mu \mathrm{g} / \mathrm{kg})$ were admixed with NPs and the resulting particles were further PEGylated with a 1:1 mixture of DSPE-PEG 2000 and DSPE$\mathrm{PEG}_{2000}$-AA. Each formulation was administered to mice once every two days via i.v administration (a). The dosage effect of MPS-cytC-NP-PEG-AA in H460 tumor model was evaluated after intravenous injection every other day. Doses for this study included 80, 160 and $320 \mu \mathrm{g} / \mathrm{kg}$ of MPS-cytC-NP-PEG-AA (P value: $*<0.05$, $* * 0.001$ ), Mean \pm SEM $(\mathrm{n}=5) .(\mathbf{b})$ 


\section{Table I}

Quantification of the percentage of H460 cells stained with annexin V and PI after 24 hour treatment with different nanoparticle formulations. Data was determined from the combined Q2 and Q4 quadrants of Figure 6 and is expressed as mean $\pm \operatorname{SEM}(\mathrm{N}=3)$.

\begin{tabular}{|l|l|}
\hline Treatment & $\mathbf{Q 2}+\mathbf{Q 4}$ \\
\hline PBS & $\mathbf{1 . 9} \pm \mathbf{0 . 8}$ \\
\hline Empty NP-PEG-AA & $\mathbf{2 . 1} \pm \mathbf{1 . 0}$ \\
\hline cytC & $\mathbf{4 . 7} \pm \mathbf{0 . 8}$ \\
\hline cytC-NP-PEG-AA & $\mathbf{2 . 2} \pm \mathbf{0 . 1}$ \\
\hline MPS-cytC & $\mathbf{1 3 . 4} \pm \mathbf{3 . 1}$ \\
\hline MPS-cytC-NP-PEG-AA & $\mathbf{2 4 . 4} \pm \mathbf{4 . 1}$ \\
\hline
\end{tabular}

